\title{
TWO-DIMENSIONAL MODELING OF MULTIFREQUENCY COMPOSITES
}

\author{
N. Lamberti, F. Montero de Espinosa*, S. Sánchez*, A.Iula and M. Pappalardo**
}

Dept. Ing. Informaz. de Ing. Elettrica, Universitá di Salerno, Fisciano I84084 (SA) Jtaly

- Instituto de Acústica, C.S.I.G., Serrano 144, 28006 Madrid, Spain

**Dept. de Ing. Elettronica,III Univ.Roma, Via C. Segre 2, 00146,Roma Italy.

\begin{abstract}
Multifrequency composites seems to be a good approach to increase the acoustic bandwidth of standard piezoelectric composites. The mechanical coupling between the different piezoceramic elements introduces new degrees of freedom into their design. Monodimensional models have been developed to study their performances in a first approximation. In order to obtain a powerful design model, a two-dimensional model used previously to describe multielement array transducers has been extended to the case of 2-2 polymer- piezoceramic composites. Several composite samples having piezoceramic strips of different width-to-thickness ratio have been built comparing their resonance behavior with the model prediction. Finally, the model has been modified to the case of 2-2 multifrequency composites. The comparison between the experimental results and the predicted ones in the case of composites with ceramic strips of two and three different lateral dimensions is presented, showing good agreement.
\end{abstract}

\section{INTRODUCTION}

Multifrequency composites have proven to be an alternative design in order to improve the frequency bandwidth of 2-2 and 1-3 piezocomposites. The basic idea is to make a composite with groups of piezoelectric elements of different lateral dimensions reproduced periodically in the structure (Figure 1).

Since the lateral and the thickness dimensions of the piezoelements in the group are of the same order of magnitude, the associate resonant modes are strongly coupled together with a consequent thickness frequency dispersion; with this technique a significant increase of the resonant bandwidth of the overall composite is obtained; further, the lack of a single periodicity reduce the propagation of unwanted spurious modes. To model the performances of these composites, a monodimensional model was previously developed (1). This model used the Onoe coupling modes theory to predict the resonance frequency of the ceramic strips in function of their width-to-thickness ratio. Then, virtual elastic constants were defined to convert ceramic strips of the same material but with different width-to-thickness ratio on ceramic strips of different virtual materials but with the same width-to-thickness ratio. Doing that, the well known monodimensional composite models could be used, being able to predict the variation of the resonance frequency of the composites in function of the lateral dimensions of the piezoceramic elements. The next step was to develop a transducer equivalent circuit model to be able to predict the transducer behavior of bi-frequency composites. The problem arise from the modeling of the coupling between different resonators in the same composite structure. Only empirical attempts based on the vibration study of these structures were possible to use ( 3 ).

In this paper we have used a bidimensional model of the vibration of both the piezoceramic strips and the polymer ones, which takes into account the lateral coupling between the composite elements. The comparison with the experimental results obtained with several composite samples shows the adequacy of the approach.

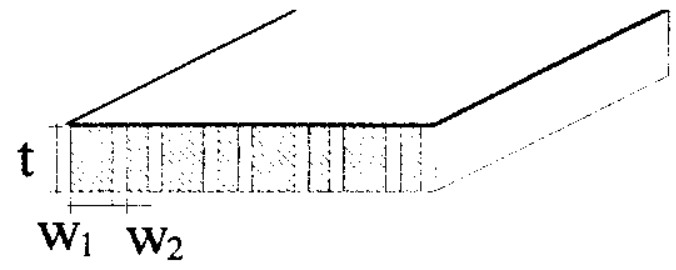

Figure 1: 2-2 multifrequency composite- double composite-

\section{2.- TRANSDUCER MODEL}

The piezoelectric element of the composite has the typical structure shown in fig. 2; its vibration in the $x$ and $z$ directions can be described, in the frequency domain, by the following matrix (2):

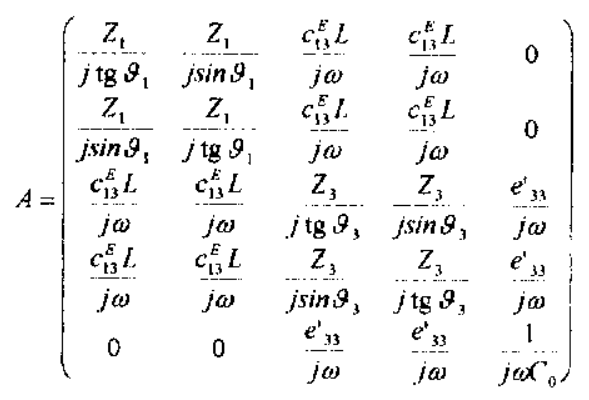

where:

$\vartheta_{1}=\frac{w \omega}{v_{1}} ; \quad \vartheta_{3}=\frac{w l}{v_{3}}$ 
being $Z_{1}$ and $Z_{3}$ the piezoceramic acoustic impedances along the $x$ and $z$ directions respectively. In this model we neglect the piezoelectric coupling in the transversal direction and satisfy the stress boundary conditions only in an integral form, but these approximations do not substantially affect the results, as it has been shown (2).

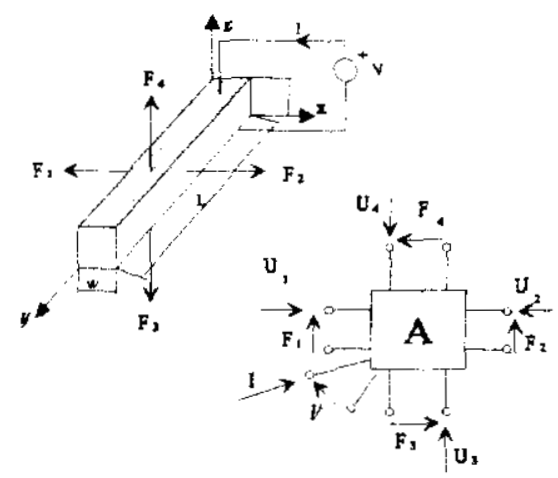

Figure 2: Geometry of the piezoceramic element of the composite

The polymer strips are also considered as a two-dimensional structure (fig.3), therefore its model can be simply deduced from that of the piezoelectric element simply cancelling all the piezoelectric constants. So they can be represented by the next matrix:

$A_{i}=\left(\begin{array}{cccc}Z_{1 i} & Z_{1 i} & c_{13} L & \frac{c_{13} L}{j \omega} \\ j \operatorname{tg} \vartheta_{1 i} & j \sin \vartheta_{1 i} & j \omega & j \omega \\ Z_{1 i} & Z_{1 i} & c_{13} L & c_{13} L \\ j \sin \vartheta_{1 i} & j \operatorname{tg} \vartheta_{1 i} & j \omega & j \omega \\ c_{13} L & c_{13} L & Z_{3 i} & Z_{3 i} \\ j \omega & j \omega & j \operatorname{tg} \vartheta_{3 i} & j \sin \vartheta_{3 i} \\ c_{13} L & c_{13} L & Z_{3 i} & Z_{3 i} \\ j \omega & j \omega & j \sin \vartheta_{3 i} & j \operatorname{tg} \vartheta_{3 i}\end{array}\right)$

where

$$
\vartheta_{l i}=\frac{w_{i} \omega}{v_{i}} ; \quad \vartheta_{3 i}=\frac{\omega l_{i}}{v_{i}}
$$

and $Z_{31}, Z_{3 i}$ are the polymer acoustic impedances along the $x$ and $z$ directions and $v_{i}$ is the velocity of the polymer.

From an electrical point of view, these polymer elements are capacitors because, in a composite, the surfaces perpendicular to the $\mathrm{z}$ axis are metallized. To take into account this situation, we inserted into the matrix the expression $1 / \omega \mathrm{C}_{0 \mathrm{j}}$ at the place $(5,5)$, being $C_{0 i j}$ the capacitance of the strip

The full standard composite can be seen as a cascade connection of the piezoelectric 5-bipole alternated to the nonpiezoelectric polymer 5-bipole (Figure 4). The connection between the ports of the multipoles represents the mechanical contact between the elements. In order to build the matrix of the overall composite, we used an algorithm which computes

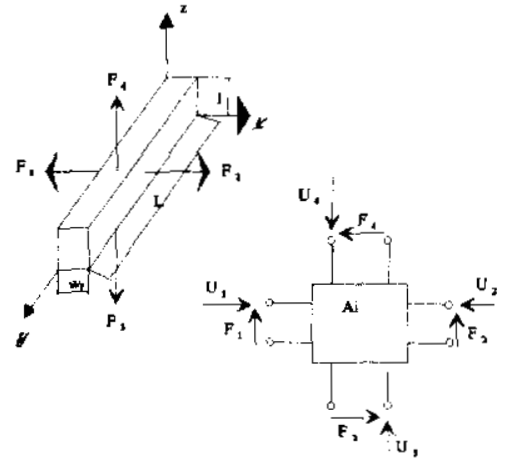

Figure 3: Geometry of the polymer composite strips

the resulting matrix of the cascade of the two elemental matrices (3). Iterating this algorithm to all the composite elements, we obtain the total matrix. We applied first this technique

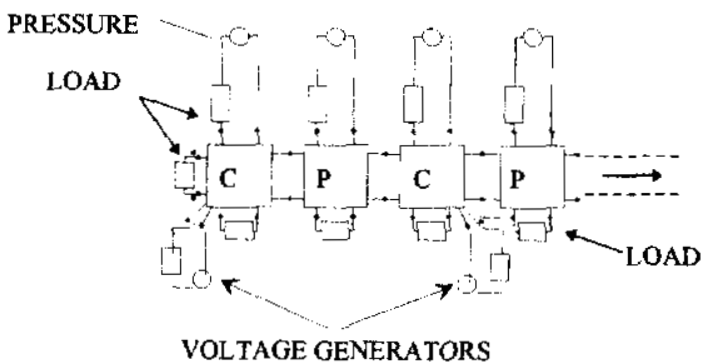

Figure 4: Block diagram of the standard composite. C, piezoceramic, $P$, polymer

to compute the matrix representing 2-2 standard composites, so, having both the polymer and the piezoelectric elements of the same dimensions. With this complete matrix, we have simulated the electrical input impedance of three standard composites, called $\mathrm{CP}, \mathrm{CM}$ and $\mathrm{CG}$, which dimensions are reported in Table 1. These dimensions were chosen to maintain the same ceramic volume percent in all samples.

Table I.- Standard composites dimensions

$\begin{array}{llll}\text { composite } & \begin{array}{l}\text { size - } \\ \mathrm{mm}\end{array} & \begin{array}{l}\text { piezoelectric strip } \\ \text { dimensions - mm }\end{array} & \begin{array}{l}\text { polymer strip } \\ \text { dimensions -mm }\end{array} \\ \mathrm{CP} & 14 \times 14 & 14 \times 0.68 \times 0.25 & 14 \times 0.68 \times 0.08 \\ \mathrm{CM} & 14 \times 14 & 14 \times 0.68 \times 0.4 & 14 \times 0.68 \times 0.11 \\ \mathrm{CG} & 14 \times 14 & 14 \times 0.68 \times 0.45 & 14 \times 0.68 \times 0.13\end{array}$

The second step was to modify the algorithm such as to be able to model the multifrequency composites. For instance, in the case of a double composite, the algorithm linked three different matrices, two corresponding to the two ceramic strips of different width and the third to the polymer section. 


\section{COMPOSITE FABRICATION.}

To check the model, several composites were made. A first set of three 2-2 piezoceramic-polymer samples were built having the dimensions shown in Table 1 . The piezoelectric material was PZ 27 (FERROPERM) and the polymer Araldit $\mathrm{H}$ (Ciba\&Geigy).

The standard dicing-and-filling technique was used. The composite main surfaces were sputtered with silver. We also built several multifrequency composites, using as piezoceramic constituents the ones previously used in the standard composites. So, we made double and triple composites following the scheme of table II. For instance, composite D4 consisted of a piezoceramic strips $14 \mathrm{~mm}$ long, $0.68 \mathrm{~mm}$ high and $0.25 \mathrm{~mm}$ wide, alternated with piezoceramic strips with the same thickness and length but $0.4 \mathrm{~mm}$ wide. In this case, to maintain the same ceramic percent in the composite, the polymer inter-space was $0.11 \mathrm{~mm}$ wide.

Table II. Description of the double -D4 and D5- and triple composite constituents.

composite
D4
D45
T

$$
\begin{gathered}
\text { constituents } \\
\mathrm{CP}+\mathrm{CM}+\ldots \\
\mathrm{CP}+\mathrm{CM}+\ldots . \\
\mathrm{CP}+\mathrm{CM}+\mathrm{CG}+\ldots .
\end{gathered}
$$

\section{COMPARISON BETWEEN COMPUTED AND EXPERIMENTAL RESULTS.}

A first check was made to ascertain the accuracy of the model. So, we first modelled the input electrical conductance of a double composite - D4- in two different cases: when the passive phase was Araldite and when it was air. The idea was to compare this prediction with the experimental results reported in reference (2). The mechanical coupling between the different piezoelectric resonators originates a complicate vibration pattern on the composite which makes impossible to model the overall behavior using monodimensional approaches. In fact, if no coupling is considered, and only a parallel electrical connection of the different piezoelectric strips is considered with a kind of overall real loading to take into account the polymer lateral loading, the conductance relative maxima of composite D4 should coincide with those of the constituents, composites $\mathrm{CP}$ and $\mathrm{CM}$. Actually, neither the frequencies nor the relative amplitudes agree with those of the standard composites. Physical explanations were proposed in (2) and (3). The prediction shown in figure 5 agrees very well with the above commented results. It must be born in mind that the two frequency maxima of the uncoupled D4 prediction correspond to the frequency resonances of the piezoceramic strips, air loaded all around.

To compare the experimental and computed results, the electrical input impedance of the built composite samples were measured with a HP Impedance Analyser $4194 \mathrm{~A}$ in air loading conditions. Figure 6 shows the real part of the admittance, G, of the standard composites $\mathrm{CP}, \mathrm{CM}$ and $\mathrm{CG}$ in function of the frequency around resonance. In the same figure, the computed impedances are superimposed to comparison. As it can be seen , the model predicts quite well the resonance frequency variation produced by the corresponding resonance frequency shift of the constituent piezoceramic strips. The agreement is also very good.

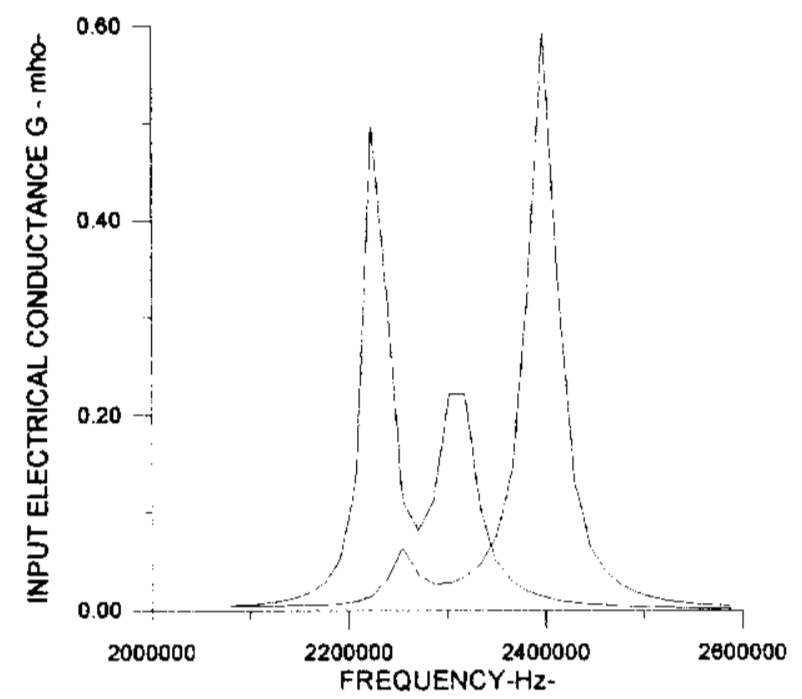

Figure 5. Comparison between predicted $\mathrm{G}$ of composite D4 when the piezoelectrc strips are coupled - Araldit in the interelement spaces - or uncoupled - air into the interelement

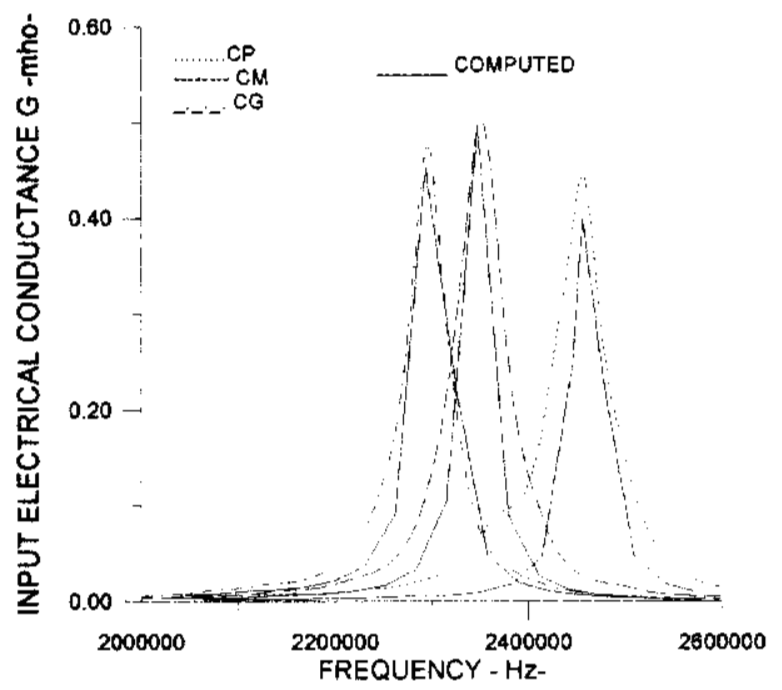

Figure 6 . Computed and measured input electrical conductance $\mathrm{G}$ of the standard composites, CP,CM, and CG.

Double and triple composites were also compared with the model outputs. Figures 7,8 and 9 show the measured conductance frequency band around resonance of the D4, D45 and $T$ composites together with their model predictions. 


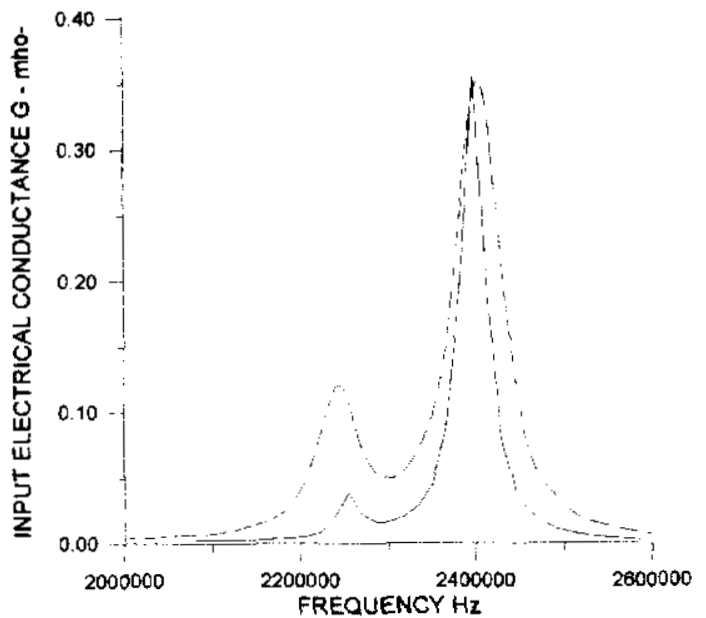

Figure 7. Conductance band of the double composite D4. Solid line, computed results, dashed line, measured results

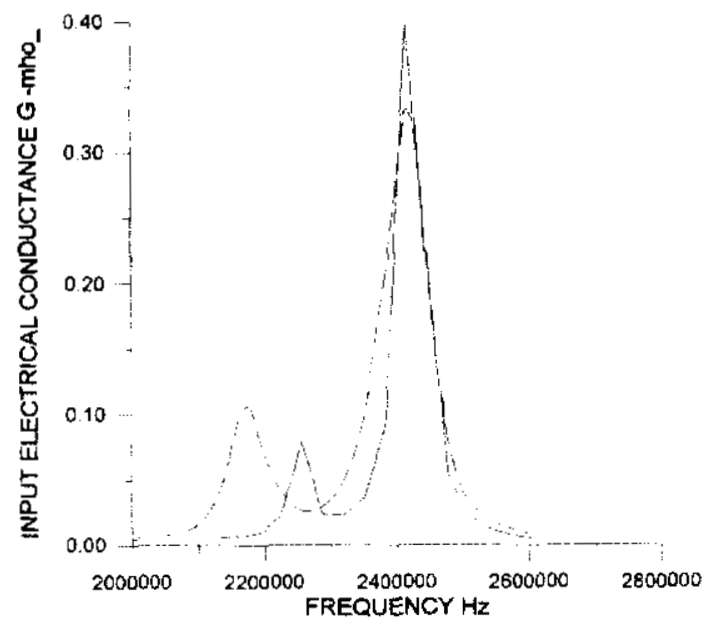

Figure 8. Conductance band of the double composite D45. Solid line , computed results, dashed line, measured results

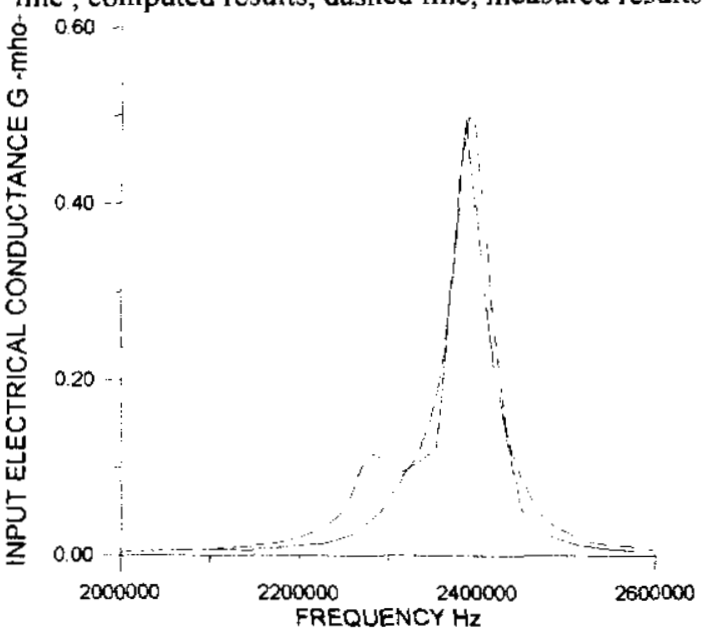

Figure 9. Conductance band of the triple composite T. Solid line, computed results, dashed line, measured results

\section{CONCLUSIONS}

A two-dimensional model of standard 2-2 piezoelectric composites has been developed as an extension of other approach applied to array transducers. The model predicts with accuracy the resonance frequency shift observed when the lateral dimensions of the piezoceramic strips are varied.

The model can also simulate the multifrequency composites, which have piezoelectric elements with different width placed alternatively along the composite.

The good agreement found between the model outputs and the measurements of the input electrical impedance of both, standard and multifrequency composites makes this model a unique tool to the design of this kind of composite structures.

\section{ACKNOWLEDGEMENTS.}

This work has been partially funded by the CDTI under the project PACE - PC144-

\section{REFERENCES. -}

1.- F.R. Montero de Espinosa, S. Sánchez, P.T. Sanz, "Modelling, construction and performance test of multifrequency composites", Acoustic Sensing and Probing, Ed A. Alippi, Word Scientific, pp 371-378, 1992.

2.- N. Lamberti, M. Pappalardo, "A two-dimensional model for bounded resonant piezoelectric ceramic plate", Proc. IEEE Ultrasonics Symposium, pp 775-779, (1989).

3.- N. Lamberti, V. Genovese, M. Pappalardo, "A twodimensional model of the multielement piezoelectric transducer", Proc. IEEE Ultrasonics Symposium, pp 785-789, (1990)

4.- F.R. Montero de Espinosa, J.C. Baboux, J. Zhang, “ Differences between coupled and uncoupled multifrequency composites", Third Euroceramics, Ed by P.Durán and J.F Fernández, Faenza Edittrice, Italia,193-198, 1993, CL. 